\title{
Copper-catalyzed asymmetric methylation of fluoroalkylated pyruvates with dimethylzinc
}

\author{
Kohsuke Aikawa, Kohei Yabuuchi, Kota Torii and Koichi Mikami
}

\author{
Full Research Paper \\ Address: \\ Department of Chemical Science and Engineering, School of \\ Materials and Chemical Technology, Tokyo Institute of Technology, \\ O-okayama, Meguro-ku, Tokyo 152-8552, Japan \\ Email: \\ Koichi Mikami* - mikami.k.ab@m.titech.ac.jp \\ * Corresponding author \\ Keywords: \\ asymmetric methylation; chiral phosphine ligand; copper catalyst; \\ dimethylzinc; trifluoropyruvate
}

\author{
Beilstein J. Org. Chem. 2018, 14, 576-582. \\ doi:10.3762/bjoc. 14.44 \\ Received: 30 October 2017 \\ Accepted: 19 February 2018 \\ Published: 07 March 2018 \\ This article is part of the Thematic Series "Organo-fluorine chemistry IV". \\ Guest Editor: D. O'Hagan \\ (C) 2018 Aikawa et al.; licensee Beilstein-Institut. \\ License and terms: see end of document.
}

\begin{abstract}
The catalytic asymmetric methylation of fluoroalkylated pyruvates is shown with dimethylzinc as a methylating reagent in the presence of a copper catalyst bearing a chiral phosphine ligand. This is the first catalytic asymmetric methylation to synthesize various $\alpha$-fluoroalkylated tertiary alcohols with $\mathrm{CF}_{3}, \mathrm{CF}_{2} \mathrm{H}, \mathrm{CF}_{2} \mathrm{Br}$, and $n-\mathrm{C}_{n} \mathrm{~F}_{2 n+1}(n=2,3,8)$ groups in good-to-high yields and enantioselectivities. Axial backbones and substituents on phosphorus atoms of chiral phosphine ligands critically influence the enantioselectivity. Moreover, the methylation of simple perfluoroalkylated ketones is found to be facilitated by only chiral phosphines without copper.
\end{abstract}

\section{Introduction}

The introduction of fluorine atoms into organic compounds plays an important role in the discovery of lead candidates with unique biological and physicochemical properties [1,2]. Therefore, the development of novel synthetic methods for the introduction of fluorinated fragments, such as trifluoromethyl $\left(\mathrm{CF}_{3}\right)$, difluoromethyl $\left(\mathrm{CF}_{2} \mathrm{H}\right)$, and difluoromethylene $\left(-\mathrm{CF}_{2}-\right)$, has attracted a great deal of attention from synthetic organic chemists [3-6]. Among these methods, many researchers including us have studied the catalytic asymmetric synthesis of optically active $\alpha$-trifluoromethylated tertiary alcohols $[7,8]$. In these cases, one of commercially available and versatile trifluoro- methyl sources, trifluoropyruvate, has been utilized for a variety of catalytic asymmetric carbon-carbon bond forming reactions, providing efficiently $\alpha$-trifluoromethylated tertiary alcohols in high enantioselectivities [9-19]. Over the past decade we have also investigated several catalytic asymmetric reactions using trifluoropyruvate as an electrophile in the presence of a chiral Lewis acid complex [20-27]. However, the synthetic method for chiral $\alpha$-trifluoromethylated tertiary alcohols via methylation of trifluoropyruvate is quite limited, although several drug candidates bearing this chiral trifluoromethylated moiety have so far been reported [7,28-30]. In 2007, Gosselin and Britton et al. re- 
ported that treatment of ethyl trifluoropyruvate (1a) with $(R)$ BINOL-mediated organozincate as a chiral methylating regent provided the corresponding methylated tertiary alcohol 2a in moderate enantioselectivity (Scheme 1, reaction 1) [31]. Kinetic resolution of racemic $\alpha$-trifluoromethylated tertiary alcohols $\mathbf{2 a}$ by an enzyme is also reported to give the corresponding alcohols 2a in high enantioselectivity (Scheme 1, reaction 2) [32]. However, there has been no report for catalytic asymmetric methylation of trifluoropyruvate. Herein, we disclose the catalytic asymmetric methylation of trifluoropyruvate derivatives as electrophiles and dimethylzinc as a methylating nucleophile by a chiral copper catalyst. This method is also applicable to the asymmetric synthesis of various $\alpha$-fluoroalkylated tertiary alcohols bearing $\mathrm{CF}_{2} \mathrm{H}, \mathrm{CF}_{2} \mathrm{Br}$, and $n-\mathrm{C}_{n} \mathrm{~F}_{2 n+1}(n=2,3,8)$ groups.

\section{Results and Discussion}

Our initial investigation was focused on the methylation of ethyl trifluoropyruvate (1a) with $\mathrm{Me}_{2} \mathrm{Zn}$ in the presence of a copper salt bearing a chiral bidentate phosphine ligand (Table 1). We were delighted to find that the reaction proceeded smoothly in the presence of CuTC (TC: 2-thiophenecarboxylate, $2.5 \mathrm{~mol} \%)$ and $(R)$-BINAP $(2.7 \mathrm{~mol} \%)$ in $\mathrm{Et}_{2} \mathrm{O}$ at $-78{ }^{\circ} \mathrm{C}$, furnishing the methylated product $\mathbf{2 a}$ in $99 \%$ yield with $38 \%$ ee (Table 1, entry 1). The effect of the $\mathrm{Cu}$ salt was also surveyed. The use of CuOAc resulted in slightly decreased enantioselectivities, and (CuOTf) $\mathrm{C}_{6} \mathrm{H}_{6}$ and $\mathrm{CuI}$ led to a racemic product (Table 1, entries 2-4). Chiral phosphine ligands instead of BINAP were further assayed with the aim of enhancing the enantioselectivity. Indeed, the investigation of the effect of axial backbones and substituents on the phosphorus atoms led to an increase in the enantioselectivity. In the case of a biphenyl backbone, MeO-BIPHEP showed the same level of enantioselectivity as BINAP, while lower enantioselectivity was obtained by SEGPHOS (Table 1, entries 5 and 6). Exploring the effect of substituents on phosphorus, DM-BINAP slightly exceeded the level attained by BINAP, although Cy-BINAP and DTBM-BINAP decreased the enantioselectivities (Table 1, entries 7-9). In sharp contrast to BINAP derivatives, DTBMSEGPHOS and DTBM-MeO-BIPHEP with extremely bulky aryl groups increased the enantioselectivities (Table 1, entries 10 and 11). Additionally, DTB-MeO-BIPHEP provided the desired alcohol in $84 \%$ yield with $60 \%$ ee (Table 1, entry 12 ). In toluene and $\mathrm{CH}_{2} \mathrm{Cl}_{2}$ as noncoordinating solvents (Table 1, entries 14 and 15), the reaction gave lower enantioselectivities, but TBME gave the best result in $90 \%$ yield and $67 \%$ ee (Table 1, entry 16). The use of methyl trifluoropyruvate (1b) instead of $\mathbf{1 a}$ resulted in a lower enantioselectivity (Table 1, entry 17). The absolute configuration of $\mathbf{2} \mathbf{b}$ was determined to be $S$ by comparison with the optical rotation of reported data [32]. The absolute configurations of other alcohol products $\mathbf{2 a}$ and $\mathbf{2 c - k}$ were tentatively assigned by analogy to $\mathbf{2 b}$.

Additionally, the reaction conditions were fine-tuned as exemplified in Table 2. It was found that reactions without $\mathrm{CuTC}$ and phosphine ligand (Table 2, entry 1) or only without phosphine ligand (Table 2, entry 2 ) provided the alcohol as a racemic mixture even at $-78{ }^{\circ} \mathrm{C}$. In contrast, the chiral product was obtained in $64 \%$ yield in the absence of a copper salt but in low enantioselectivity (Table 2, entry 3 ). Therefore, decreasing the amount of phosphine ligand $(2.4 \mathrm{~mol} \%)$ to less than that of

asymmetric methylation with chiral BINOL-zincate [31]

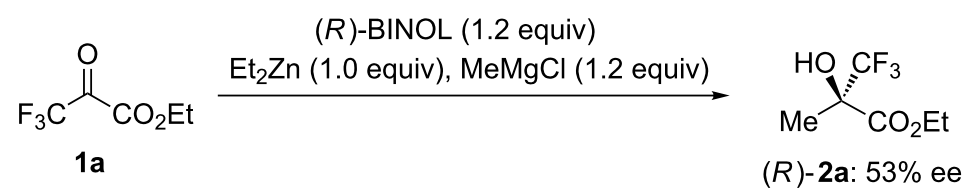

kinetic resolution of racemic substrate with enzyme [32]

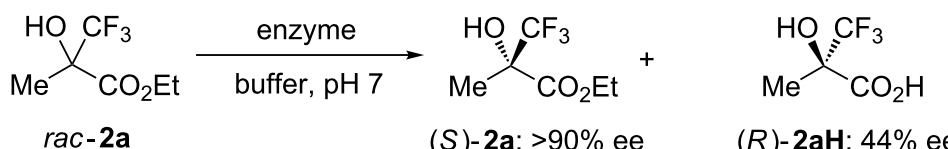

his work: catalytic asymmetric methylation with chiral copper complex

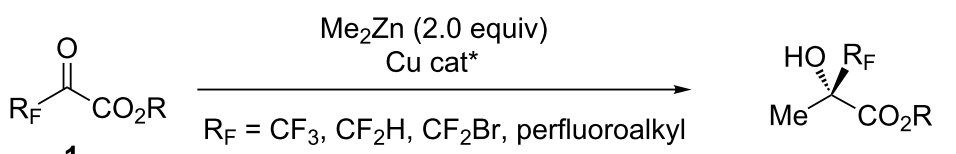

(S)-2: up to $94 \%$ ee 
Table 1: Copper-catalyzed asymmetric methylation

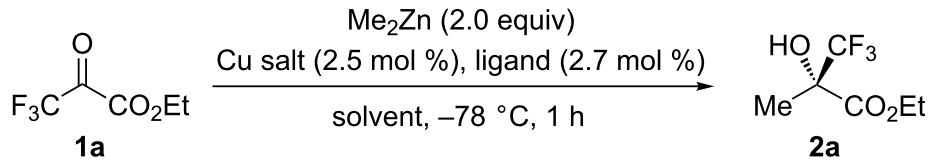

\begin{tabular}{|c|c|c|c|c|c|}
\hline entry & ligand & Cu salt & solvent & yield $(\%)^{a}$ & ee $(\%)$ \\
\hline 1 & $(R)$-BINAP & CuTC & $\mathrm{Et}_{2} \mathrm{O}$ & 99 & 38 \\
\hline 2 & $(R)$-BINAP & CuOAc & $\mathrm{Et}_{2} \mathrm{O}$ & 43 & 36 \\
\hline 3 & $(R)$-BINAP & (CuOTf) $\cdot \mathrm{C}_{6} \mathrm{H}_{6}$ & $\mathrm{Et}_{2} \mathrm{O}$ & 13 & 0 \\
\hline 4 & $(R)$-BINAP & Cul & $\mathrm{Et}_{2} \mathrm{O}$ & 38 & 0 \\
\hline 5 & $(R)$-SEGPHOS & CuTC & $\mathrm{Et}_{2} \mathrm{O}$ & 81 & 26 \\
\hline 6 & (R)-MeO-BIPHEP & CuTC & $\mathrm{Et}_{2} \mathrm{O}$ & 85 & 38 \\
\hline 7 & $(R)-\mathrm{Cy}-\mathrm{BINAP}$ & CuTC & $\mathrm{Et}_{2} \mathrm{O}$ & 92 & 3 \\
\hline 8 & $(R)$-DM-BINAP & CuTC & $\mathrm{Et}_{2} \mathrm{O}$ & 70 & 41 \\
\hline 9 & (R)-DTBM-BINAP & CuTC & $\mathrm{Et}_{2} \mathrm{O}$ & 73 & 17 \\
\hline 10 & (R)-DTBM-SEGPHOS & CuTC & $\mathrm{Et}_{2} \mathrm{O}$ & 67 & 55 \\
\hline 11 & $(R)$-DTBM-MeO-BIPHEP & CuTC & $\mathrm{Et}_{2} \mathrm{O}$ & 99 & 50 \\
\hline 12 & $(R)$-DTB-MeO-BIPHEP & CuTC & $\mathrm{Et}_{2} \mathrm{O}$ & 84 & 60 \\
\hline 13 & $(R)$-DTB-MeO-BIPHEP & CuTC & THF & 85 & 57 \\
\hline 14 & $(R)$-DTB-MeO-BIPHEP & CuTC & toluene & 98 & 38 \\
\hline 15 & $(R)$-DTB-MeO-BIPHEP & CuTC & $\mathrm{CH}_{2} \mathrm{Cl}_{2}$ & 90 & 9 \\
\hline 16 & $(R)$-DTB-MeO-BIPHEP & CuTC & TBME & 90 & 67 \\
\hline $17^{\mathrm{b}}$ & (R)-DTB-MeO-BIPHEP & CuTC & TBME & 71 & $59(S)$ \\
\hline
\end{tabular}

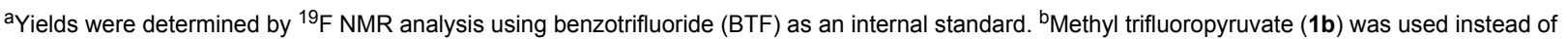
ethyl trifluoropyruvate (1a).<smiles></smiles>

(R)-BINAP<smiles>c1ccc(-c2ccc3c(c2-c2ccccc2)OCO3)cc1</smiles>

(R)-SEGPHOS<smiles>COc1cccc(-c2ccccc2)c1-c1c(OC)cccc1-c1ccccc1</smiles>

(R)-MeO-BIPHEP<smiles>CC(C)Pc1ccc2ccccc2c1-c1c(PC2CC3CCC(CC3)C2)ccc2ccccc12</smiles>

(R)-Cy-BINAP<smiles>CPc1ccc2ccccc2c1-c1c(Pc2cc(C)cc(C)c2)ccc2ccccc12</smiles>

(R)-DM-BINAP

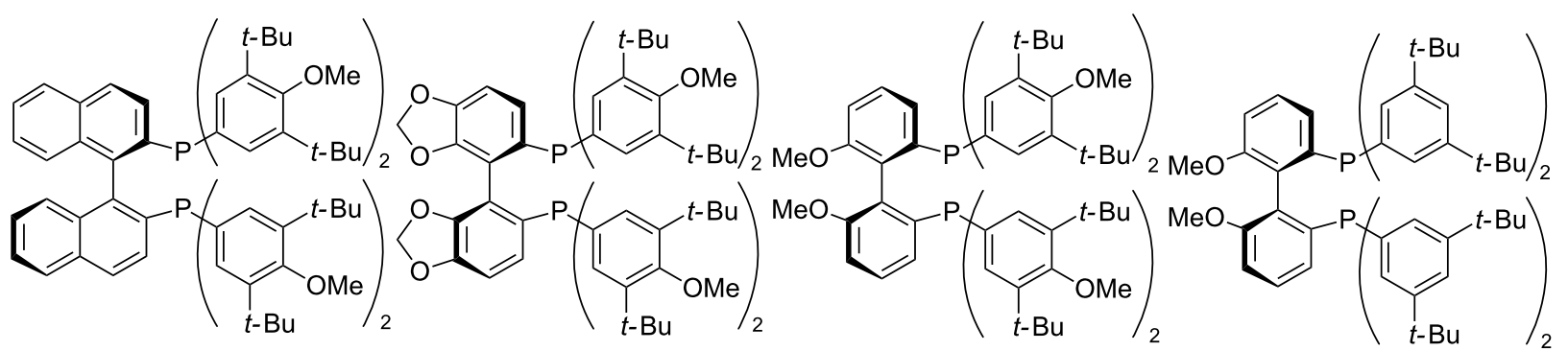

(R)-DTBM-BINAP

(R)-DTBM-SEGPHOS

(R)-DTBM-MeO-BIPHEP

(R)-DTB-MeO-BIPHEP

copper salt led to an enhancement of the enantioselectivity to $70 \%$ ee (Table 2 , entries 4 vs 5 ). In addition, the selection of BTFM-Garphos instead of DTB-MeO-BIPHEP afforded a higher enantoselectivity (Table 2, entry 6), and consequently, a lower reaction temperature $\left(-90{ }^{\circ} \mathrm{C}\right)$ gave the best result with $86 \%$ yield and $89 \%$ ee (Table 2 , entry 7 ).
Various fluoroalkylated pyruvates were applicable to this catalytic transformation under the optimized reaction conditions (Scheme 2). Alkyl substituents on the ester moiety of the trifluoropyruvate were found to influence the stereoselectivity drastically. The reaction of trifluoropyruvates $(\mathbf{1} \mathbf{c}-\mathbf{e})$ bearing sterically demanding substituents such as isopropyl, cyclopentyl, 
Table 2: Optimization of reaction conditions.

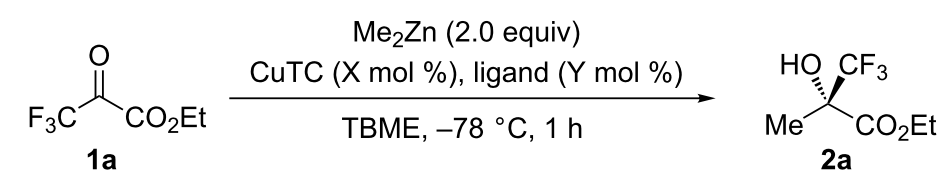

\begin{tabular}{|c|c|c|c|c|c|}
\hline entry & $\mathrm{X}(\mathrm{mol} \% / \mathrm{Cu})$ & Y (mol \%/ligand) & ligand & yield $(\%)^{a}$ & ee $(\%)$ \\
\hline 1 & 0 & 0 & - & 17 & - \\
\hline 2 & 2.5 & 0 & - & 15 & - \\
\hline 3 & 0 & 2.5 & $(R)$-DTB-MeO-BIPHEP & 64 & 7 \\
\hline 4 & 2.5 & 2.7 & (R)-DTB-MeO-BIPHEP & 90 & 67 \\
\hline 5 & 2.5 & 2.4 & (R)-DTB-MeO-BIPHEP & 94 & 70 \\
\hline 6 & 2.5 & 2.4 & (R)-BTFM-Garphos & 88 & 73 \\
\hline $7^{\mathrm{b}}$ & 2.5 & 2.4 & (R)-BTFM-Garphos & 86 & 89 \\
\hline
\end{tabular}

ayields were determined by ${ }^{19} \mathrm{~F}$ NMR analysis using benzotrifluoride (BTF) as an internal standard. ${ }^{\mathrm{b}}$ Reaction temperature was $-90{ }^{\circ} \mathrm{C}$.<smiles>COc1cc(OC)c(-c2c(OC)cc(OC)cc2Pc2cc(C(F)(F)F)cc(C(F)(F)F)c2)c(Pc2cc(C(F)(F)F)cc(C(F)(F)F)c2)c1</smiles>

(R)-BTFM-Garphos

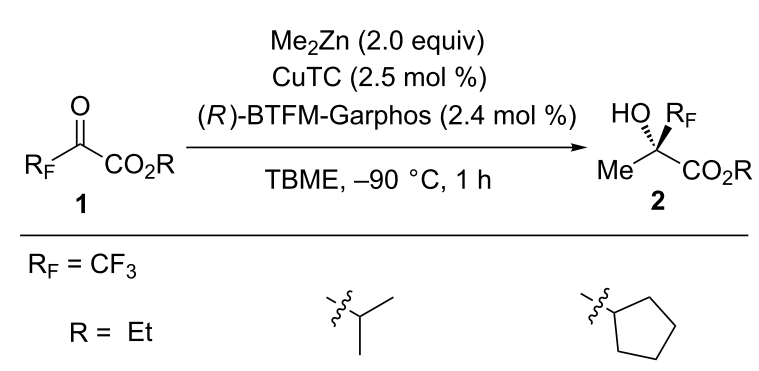

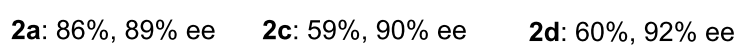<smiles>CC1CCCCC1</smiles><smiles>CC(C)C(C)C(C)C</smiles>

2e: $64 \%, 94 \%$ ee $\quad$ 2f: $71 \%, 64 \%$ ee

$$
\mathrm{R}=\mathrm{Et}
$$$$
\mathrm{R}_{\mathrm{F}}=\mathrm{CF}_{2} \mathrm{H}
$$

$\mathrm{CF}_{2} \mathrm{Br}$

$\mathrm{C}_{2} \mathrm{~F}_{5}$

2g: $89 \%, 89 \%$ ee $\quad$ 2h: $53 \%, 82 \%$ ee $^{\text {a }} \quad \mathbf{2 i}: 87 \%, 86 \%$ ee

$$
n-\mathrm{C}_{3} \mathrm{~F}_{7} \quad n-\mathrm{C}_{8} \mathrm{~F}_{17}
$$

2j: $98 \%, 78 \%$ ee $\quad 2 k: 92 \%, 73 \%$ ee

Scheme 2: Scope of fluoroalkylated pyruvates. Yields were determined by ${ }^{19} \mathrm{~F}$ NMR analysis using benzotrifluoride (BTF) as an internal standard. aReaction temperature was $-78^{\circ} \mathrm{C}$. and cyclohexyl led to a higher level of enantioselectivities (90-94\% ee), compared to the corresponding ethyl ester 1a. In contrast, trifluoropyruvate $\mathbf{1 f}$ with an extremely bulky substituent caused a decrease of enantioselectivity. Ethyl difluoropyruvate (1g) and ethyl bromodifluoropyruvate (1h) also underwent the reactions in good enantioselectivities, although a slight decrease in yield was observed due to the steric hindrance of the $\mathrm{CF}_{2} \mathrm{Br}$ group. Significantly, ethyl perfluoropyruvates $\mathbf{1} \mathbf{i}-\mathbf{k}$ with longer alkyl chains were also converted to the desired tertiary alcohols in good enantioselectivities.

The catalytic asymmetric methylation using the simple perfluoroalkylated ketone 3a instead of pyruvate derivatives was further examined (Scheme 3). In contrast to the pyruvate system, the combination of CuTC and BINAP did not facilitate the reaction even at $-78{ }^{\circ} \mathrm{C}$, but also afforded the racemic product ( $25 \%$ yield, $0 \%$ ee). Interestingly, the use of only BINAP without CuTC led to higher reactivity and enantioselectivity (54\% yield, $8 \%$ ee), while BTFM-Garphos decreased the reactivity (7\% yield, $8 \%$ ee). After screening of phosphines, DTBMeO-BIPHEP was found to smoothly catalyze the asymmetric methylation to give the desired alcohol $4 \mathbf{a}$ in $87 \%$ yield and $24 \%$ ee. 


\section{$\mathrm{Me}_{2} \mathrm{Zn}$ (2.0 equiv) CuTC (5 mol \%)

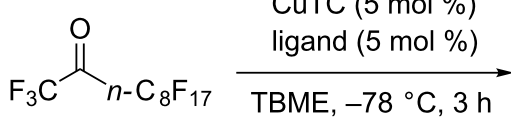 $3 a$ \\ (R)-BINAP: $25 \%, 0 \%$ ee (R)-BINAPa: $54 \%$, $8 \%$ ee (R)-BTFM-Garphos ${ }^{\text {a: }}$ : 7\%, $8 \%$ ee $(R)$-DTB-MeO-BIPHEPa: $87 \%$, 24\% ee}

Scheme 3: Catalytic asymmetric methylation of the simple perfluoroalkylated ketone 3a. Yields were determined by ${ }^{19} \mathrm{~F}$ NMR analysis using benzotrifluoride (BTF) as an internal standard. ${ }^{a}$ Reaction was carried out without CuTC.

\section{Conclusion}

In summary, we have succeeded in the catalytic enantioselective methylation of fluoroalkylated pyruvates in the presence of chiral diphosphine-copper complexes, providing the corresponding tertiary alcohols with an $\mathrm{R}_{\mathrm{F}}$ group such as $\mathrm{CF}_{3}, \mathrm{CF}_{2} \mathrm{H}$, $\mathrm{CF}_{2} \mathrm{Br}$, and $n-\mathrm{C}_{n} \mathrm{~F}_{2 n+1}(n=2,3,8)$ in good-to-high yields and enantioselectivities. This is the first report on catalytic asymmetric methylation with fluoroalkylated pyruvates. Moreover, a simple perfluoroalkyl ketone was also found to be methylated enantioselectively with dimethylzinc and a catalytic amount of a chiral diphosphine, but without copper.

\section{Experimental}

Typical procedure for copper-catalyzed asymmetric methylation of fluoroalkylated pyruvates: To a mixture of $\mathrm{CuTC}$ $(1.0 \mathrm{mg}, 0.005 \mathrm{mmol})$ and $(R)$-BTFM-Garphos $(5.7 \mathrm{mg}$, $0.0048 \mathrm{mmol})$ was added $\mathrm{CH}_{2} \mathrm{Cl}_{2}(1.0 \mathrm{~mL})$ at room temperature under an argon atmosphere, and the solution was stirred for $12 \mathrm{~h}$. The solvent was removed under reduced pressure, and the prepared catalyst was dissolved in TBME $(0.5 \mathrm{~mL})$ under an argon atmosphere. After the solution was cooled to $-90{ }^{\circ} \mathrm{C}$, $\mathrm{Me}_{2} \mathrm{Zn}$ (1.0 M in heptane, $\left.0.4 \mathrm{~mL}, 0.4 \mathrm{mmol}\right)$ followed by fluoroalkylated pyruvate $1(0.2 \mathrm{mmol})$ in TBME $(0.5 \mathrm{~mL})$ were added over $30 \mathrm{~min}$. The reaction mixture was stirred at the same temperature for $1 \mathrm{~h}$. The reaction mixture was quenched with saturated aq $\mathrm{NH}_{4} \mathrm{Cl}$ solution. The organic layer was separated and the aqueous layer was extracted twice with $\mathrm{Et}_{2} \mathrm{O}$. The combined organic layer was dried over anhydrous $\mathrm{Na}_{2} \mathrm{SO}_{4}$ and evaporated under reduced pressure $(350 \mathrm{mmHg})$. The concentrated solution was used without purification for the next protection reaction. The yield of alcohol product 2 was determined by ${ }^{19} \mathrm{~F}$ NMR analysis using benzotrifluoride (BTF) as an internal standard.

To a solution of DMAP $(2.4 \mathrm{mg}, 0.02 \mathrm{mmol})$ and the crude alcohol 2 in $\mathrm{CH}_{2} \mathrm{Cl}_{2}(2.0 \mathrm{~mL})$ was added $\mathrm{NEt}_{3}(56 \mu \mathrm{L}$, $0.4 \mathrm{mmol}$ ) at room temperature under an argon atmosphere.
After the reaction mixture was cooled to $0{ }^{\circ} \mathrm{C}, p$-nitrobenzoyl chloride (56 mg, $0.3 \mathrm{mmol}$ ) was added. Then the mixture was warmed to room temperature and stirred for $1 \mathrm{~h}$. After $1 \mathrm{~N} \mathrm{HCl}$ $(5.0 \mathrm{~mL})$ was added to the reaction mixture, the organic layer was separated and the aqueous layer was extracted twice with $\mathrm{Et}_{2} \mathrm{O}$. The combined organic layer was washed with saturated aq. $\mathrm{NaHCO}_{3}$, water, and brine, and then dried over anhydrous $\mathrm{MgSO}_{4}$ and evaporated under reduced pressure. The residue was purified by silica gel column chromatography to give $p$-nitrobenzoylated alcohol 2'. The enantiomeric excess was determined by chiral HPLC analysis.

\section{(S)-3-Ethoxy-1,1,1-trifluoro-2-methyl-3- oxopropan-2-yl 4-nitrobenzoate (2a')}

The yield of alcohol 2a (86\%) was determined by ${ }^{19} \mathrm{~F}$ NMR analysis. $p$-Nitrobenzoylated alcohol 2a' was purified by silicagel column chromatography (EtOAc/hexane 1:40) as a colorless liquid (53\% yield for 2 steps, $89 \%$ ee). ${ }^{1} \mathrm{H}$ NMR (300 MHz, $\left.\mathrm{CDCl}_{3}\right) \delta 8.34-8.31(\mathrm{~m}, 2 \mathrm{H}), 8.24-8.20(\mathrm{~m}, 2 \mathrm{H}), 4.33(\mathrm{q}, 4 \mathrm{H}, J$ $=6.9 \mathrm{~Hz}), 1.97(\mathrm{~d}, 3 \mathrm{H}, J=0.9 \mathrm{~Hz}), 1.28(\mathrm{t}, 3 \mathrm{H}, J=7.0 \mathrm{~Hz})$; ${ }^{13} \mathrm{C}$ NMR $\left(75 \mathrm{MHz}, \mathrm{CDCl}_{3}\right) \delta 164.3,162.3,151.1,134.0$, $131.2,123.7,122.7\left(\mathrm{q}, J_{\mathrm{C}-\mathrm{F}}=282.9 \mathrm{~Hz}\right), 80.7\left(\mathrm{q}, J_{\mathrm{C}-\mathrm{F}}=30.4\right.$ $\mathrm{Hz}), 63.2,16.6,13.8 ;{ }^{19} \mathrm{~F}$ NMR $\left(282 \mathrm{MHz}, \mathrm{CDCl}_{3}\right) \delta-78.4(\mathrm{~s}$, $3 \mathrm{~F}$ ); HRMS (APCI-TOF): $[\mathrm{M}]^{-\cdot}$ calcd for $\mathrm{C}_{13} \mathrm{H}_{12} \mathrm{~F}_{3} \mathrm{NO}_{6}$, 335.0617; found, 335.0623; FTIR (neat, $\mathrm{cm}^{-1}$ ) 784, 813, 849, $876,927,1011,1109,1149,1273,1342,1387,1452,1525$, $1602,1740,1763,2857,2920,2952,2996,3087,3116 ;[\alpha]_{\mathrm{D}}{ }^{22}$ -28.94 ( c 0.20, $\mathrm{CHCl}_{3}$ ); HPLC (column, CHIRALCEL OJ-3, hexane/2-propanol 91:9, flow rate $0.6 \mathrm{~mL} / \mathrm{min}, 20{ }^{\circ} \mathrm{C}$ detection UV $254 \mathrm{~nm}) t_{\mathrm{R}}$ of major isomer $13.1 \mathrm{~min}, t_{\mathrm{R}}$ of minor isomer $23.8 \mathrm{~min}$.

\section{(S)-1-Ethoxy-3,3-difluoro-2-methyl-1- oxopropan-2-yl benzoate (2 $\mathbf{g}^{\text {') }}$}

Reaction temperature was $-78^{\circ} \mathrm{C}$. The yield of alcohol $\mathbf{2 g}$ $(89 \%)$ was determined by ${ }^{19} \mathrm{~F}$ NMR analysis. In the protection of alcohol, benzoyl chloride was used instead of $p$-nitrobenzoyl chloride. Benzoylated alcohol 29' was purified by silica gel column chromatography (EtOAc/hexane 1:40) as a colorless liquid ( $41 \%$ yield for 2 steps, $89 \%$ ee). ${ }^{1} \mathrm{H}$ NMR (300 MHz, $\left.\mathrm{CDCl}_{3}\right) \delta 8.05(\mathrm{dd}, J=8.3,1.3 \mathrm{~Hz}, 2 \mathrm{H}) .7 .61(\mathrm{tt}, J=6.7,1.3$ $\mathrm{Hz}, 1 \mathrm{H}), 7.49-7.44(\mathrm{~m}, 2 \mathrm{H}), 6.30\left(\mathrm{dd}, J_{\mathrm{H}-\mathrm{F}}=56.8,54.8 \mathrm{~Hz}\right.$, $1 \mathrm{H}), 4.29(\mathrm{q}, J=7.1 \mathrm{~Hz}, 2 \mathrm{H}), 1.77\left(\mathrm{t}, J_{\mathrm{H}-\mathrm{F}}=1.6 \mathrm{~Hz}, 3 \mathrm{H}\right), 1.27$ $(\mathrm{t}, J=7.1 \mathrm{~Hz}, 3 \mathrm{H}) ;{ }^{13} \mathrm{C} \mathrm{NMR}\left(75 \mathrm{MHz}, \mathrm{CDCl}_{3}\right) \delta 167.6,164.8$, $133.7,130.0,128.8,128.5,122.9\left(\mathrm{dd}, J_{\mathrm{C}-\mathrm{F}}=250.0,245.0 \mathrm{~Hz}\right)$, $79.7\left(\mathrm{dd}, J_{\mathrm{C}-\mathrm{F}}=27.5,21.9 \mathrm{~Hz}\right), 62.3,14.6\left(\mathrm{t}, J_{\mathrm{C}-\mathrm{F}}=3.2 \mathrm{~Hz}\right)$ $13.9 ;{ }^{19} \mathrm{~F}$ NMR $\left(282 \mathrm{MHz}, \mathrm{CDCl}_{3}\right) \delta-128.40(\mathrm{dd}, J=290.2$ $\left.\mathrm{Hz}, J_{\mathrm{F}-\mathrm{H}}=54.7 \mathrm{~Hz}, 1 \mathrm{~F}\right),-132.76\left(\mathrm{dd}, J=289.90 \mathrm{~Hz}, J_{\mathrm{F}-\mathrm{H}}=\right.$ $56.4 \mathrm{~Hz}, 1 \mathrm{~F}$ ); HRMS (APCI-TOF): $[\mathrm{M}+\mathrm{Na}]^{+}$calcd for $\mathrm{C}_{13} \mathrm{H}_{14} \mathrm{~F}_{2} \mathrm{NaO}_{4}, 295.0758$; found, 295.0761; FTIR (neat, $\mathrm{cm}^{-1}$ ) 1026, 1093, 1114, 1216, 1279, 1388, 1452, 1602, 1730, 1747, 
2938, 2985, 3021; $[\alpha]_{\mathrm{D}}{ }^{25}-7.47\left(c 1.01, \mathrm{CHCl}_{3}\right)$; HPLC (column, CHIRALCEL OJ-3, hexane/2-propanol 99:1, flow rate $0.6 \mathrm{~mL} / \mathrm{min}, 20^{\circ} \mathrm{C}$ detection UV $220 \mathrm{~nm}$ ) $t_{\mathrm{R}}$ of major isomer $21.2 \mathrm{~min}, t_{\mathrm{R}}$ of minor isomer $22.6 \mathrm{~min}$.

\section{(S)-1-Bromo-3-ethoxy-1,1-difluoro-2-methyl- 3-oxopropan-2-yl 4-nitrobenzoate (2h')}

Reaction temperature was $-78^{\circ} \mathrm{C}$. The yield of alcohol $\mathbf{2 h}$ (53\%) was determined by ${ }^{19} \mathrm{~F}$ NMR analysis. $p$-Nitrobenzoylated alcohol $\mathbf{2 h}$ ' was purified by silica gel column chromatography (EtOAc/hexane 1:50) as a white solid (32\% yield for 2 steps, $82 \%$ ee). ${ }^{1} \mathrm{H}$ NMR (300 MHz, $\left.\mathrm{CDCl}_{3}\right) \delta 8.34-8.31(\mathrm{~m}$, $2 \mathrm{H}), 8.25-8.21(\mathrm{~m}, 2 \mathrm{H}), 4.32$ (q, 2H, $J=7.2 \mathrm{~Hz}), 2.02(\mathrm{~s}, 3 \mathrm{H})$, $1.29(\mathrm{t}, 3 \mathrm{H}, J=7.0 \mathrm{~Hz}) ;{ }^{13} \mathrm{C}$ NMR $\left(75 \mathrm{MHz}, \mathrm{CDCl}_{3}\right) \delta 164.3$, $162.4,151.2,134.3,131.3,123.9,121.0\left(\mathrm{t}, J_{\mathrm{C}-\mathrm{F}}=311.6 \mathrm{~Hz}\right)$, $84.8\left(\mathrm{dd}, J_{\mathrm{C}-\mathrm{F}}=25.6,23.4 \mathrm{~Hz}\right), 63.4,18.4,14.0 ;{ }^{19} \mathrm{~F}$ NMR $\left(282 \mathrm{MHz}, \mathrm{CDCl}_{3}\right) \delta-56.9(\mathrm{~d}, 1 \mathrm{~F}, J=168.6 \mathrm{~Hz}),-58.9(\mathrm{~d}, 1 \mathrm{~F}$, $J=165.3 \mathrm{~Hz}$ ); HRMS (APCI-TOF): $[\mathrm{M}]^{-}$calcd for $\mathrm{C}_{13} \mathrm{H}_{12} \mathrm{BrF}_{2} \mathrm{NO}_{6}$, 394.9816; found, 394.9835; FTIR ( $\mathrm{KBr}$ pellet, $\mathrm{cm}^{-1}$ ) 716, 843, 876, 961, 1020, 1106, 1146, 1280, 1347, $1446,1528,1610,1751,2866,2936,2988 ;[\alpha]_{\mathrm{D}}{ }^{22}-11.99(c$ $1.55, \mathrm{CHCl}_{3}$ ); HPLC (column, CHIRALCEL OD-3, hexane/2propanol $91: 9$, flow rate $0.6 \mathrm{~mL} / \mathrm{min}, 20{ }^{\circ} \mathrm{C}$ detection $\mathrm{UV}$ $254 \mathrm{~nm}) t_{\mathrm{R}}$ of major isomer $18.2 \mathrm{~min}, t_{\mathrm{R}}$ of minor isomer $12.5 \mathrm{~min}$.

\section{(S)-1-Ethoxy-3,3,4,4,4-pentafluoro-2-methyl- 1-oxobutan-2-yl $p$-nitrobenzoate (2i')}

The yield of alcohol $\mathbf{2 i}(87 \%)$ was determined by ${ }^{19} \mathrm{~F}$ NMR analysis. $p$-Nitrobenzoylated alcohol $\mathbf{2} \mathbf{i}$ ' was purified by silica gel column chromatography (EtOAc/hexane 1:40) as a white solid (48\% yield for 2 steps, $86 \%$ ee). ${ }^{1} \mathrm{H}$ NMR (300 MHz, $\left.\mathrm{CDCl}_{3}\right) \delta 8.35-8.30(\mathrm{~m}, 2 \mathrm{H}) 8.21-8.16(\mathrm{~m}, 2 \mathrm{H}), 4.37-4.27(\mathrm{~m}$, $2 \mathrm{H}), 2.04\left(\mathrm{q}, J_{\mathrm{H}-\mathrm{F}}=0.6 \mathrm{~Hz}, 3 \mathrm{H}\right), 1.28(\mathrm{t}, J=7.1 \mathrm{~Hz}, 3 \mathrm{H})$; ${ }^{13} \mathrm{C}$ NMR $\left(75 \mathrm{MHz}, \mathrm{CDCl}_{3}\right) \delta 164.2,162.2\left(\mathrm{~d}, J_{\mathrm{C}-\mathrm{F}}=2.0 \mathrm{~Hz}\right)$, $151.0,134.1,131.0,123.8,118.6$ (qt, $J_{\mathrm{C}-\mathrm{F}}=286.1,35.6 \mathrm{~Hz}$ ), $112.0\left(\mathrm{tq}, J_{\mathrm{C}-\mathrm{F}}=263.0,36.8 \mathrm{~Hz}\right), 81.3\left(\mathrm{t}, J_{\mathrm{C}-\mathrm{F}}=25.4 \mathrm{~Hz}\right), 63.3$, 16.6, 13.7; ${ }^{19} \mathrm{~F}$ NMR $\left(282 \mathrm{MHz}, \mathrm{CDCl}_{3}\right) \delta-79.19(\mathrm{~s}, 3 \mathrm{~F})$, $-121.42(\mathrm{~d}, J=280.9 \mathrm{~Hz}, 1 \mathrm{~F}),-122.98$ (d, $J=279.7 \mathrm{~Hz}, 1 \mathrm{~F})$; HRMS (APCI-TOF): [M] $]^{-*}$ calcd for $\mathrm{C}_{14} \mathrm{H}_{12} \mathrm{~F}_{5} \mathrm{NO}_{6}, 385.0585$; found, 385.0582; FTIR ( $\mathrm{KBr}$ pellet, $\mathrm{cm}^{-1}$ ) 1014, 1142, 1208, $1222,1281,1350,1385,1533,1747,2942,2987,3059 ;[\alpha]_{\mathrm{D}}{ }^{25}$ -27.75 (c 1.02, $\mathrm{CHCl}_{3}$ ); HPLC (column, CHIRALCEL OJ-3, hexane/2-propanol 99:1, flow rate $0.6 \mathrm{~mL} / \mathrm{min}, 20^{\circ} \mathrm{C}$ detection UV $220 \mathrm{~nm}$ ) $t_{\mathrm{R}}$ of major isomer $16.2 \mathrm{~min}, t_{\mathrm{R}}$ of minor isomer $31.4 \mathrm{~min}$.

\section{(S)-1-Ethoxy-3,3,4,4,5,5,5-heptafluoro-2-} methyl-1-oxopentan-2-yl p-nitrobenzoate (2j') The yield of alcohol $\mathbf{2 j}$ (98\%) was determined by ${ }^{19} \mathrm{~F}$ NMR analysis. $p$-Nitrobenzoylated alcohol $\mathbf{2} \mathbf{j}$ ' was purified by silica- gel column chromatography (EtOAc/hexane 1:50) as a colorless oil (48\% yield for 2 steps, $78 \%$ ee). ${ }^{1} \mathrm{H}$ NMR $(300 \mathrm{MHz}$, $\left.\mathrm{CDCl}_{3}\right) \delta 8.35-8.31(\mathrm{~m}, 2 \mathrm{H}) 8.21-8.16(\mathrm{~m}, 2 \mathrm{H}), 4.36-4.29(\mathrm{~m}$, $2 \mathrm{H}), 2.07\left(\mathrm{q}, J_{\mathrm{H}-\mathrm{F}}=1.3 \mathrm{~Hz}, 3 \mathrm{H}\right), 1.28(\mathrm{t}, J=7.1 \mathrm{~Hz}, 3 \mathrm{H})$; ${ }^{13} \mathrm{C} \mathrm{NMR}\left(75 \mathrm{MHz}, \mathrm{CDCl}_{3}\right) \delta 164.2162 .2,151.0,134.1,131.0$, $123.8,117.6\left(\mathrm{qt}, J_{\mathrm{C}-\mathrm{F}}=286.9,33.9 \mathrm{~Hz}\right), 113.6\left(\mathrm{tt}, J_{\mathrm{C}-\mathrm{F}}=263.9\right.$, $30.9 \mathrm{~Hz}), 122.9\left(\mathrm{tq}, J_{\mathrm{C}-\mathrm{F}}=266.9,37.4 \mathrm{~Hz}\right), 82.2\left(\mathrm{t}, J_{\mathrm{C}-\mathrm{F}}=25.7\right.$ $\mathrm{Hz}), 63.4,16.8,13.7 ;{ }^{19} \mathrm{~F}$ NMR $\left(282 \mathrm{MHz}, \mathrm{CDCl}_{3}\right) \delta-80.65$ $(\mathrm{m}, 3 \mathrm{~F}),-117.75(\mathrm{~d}, J=288.5 \mathrm{~Hz}, 1 \mathrm{~F}),-119.60(\mathrm{~d}, J=288.2$ $\mathrm{Hz}, 1 \mathrm{~F}),-123.882$ (s, 2F); HRMS (APCI-TOF): [M] $]^{-}$calcd for $\mathrm{C}_{15} \mathrm{H}_{12} \mathrm{~F}_{7} \mathrm{NO}_{6}$, 435.0553; found, 435.0547; FTIR (neat, $\mathrm{cm}^{-1}$ ) 1090, 1140, 1200, 1233, 1349, 1387, 1534, 1609, 1744, 1761, 2942, 2988, 3059; $[\alpha]_{\mathrm{D}}{ }^{25}-22.60\left(c 0.94, \mathrm{CHCl}_{3}\right)$; HPLC (column, CHIRALCEL OJ-3, hexane/2-propanol 99:1, flow rate $0.6 \mathrm{~mL} / \mathrm{min}, 20^{\circ} \mathrm{C}$ detection UV $220 \mathrm{~nm}$ ) $t_{\mathrm{R}}$ of major isomer $12.2 \mathrm{~min}, t_{\mathrm{R}}$ of minor isomer $15.5 \mathrm{~min}$.

(S)-1-Ethoxy-3,3,4,4,5,5,6,6,7,7,8,8,9,9,10, 10,10-heptadecafluoro-2-methyl-1-oxodecan2-yl p-nitrobenzoate (3k')

The yield of alcohol $\mathbf{2 k}$ (92\%) was determined by ${ }^{19} \mathrm{~F}$ NMR analysis. $p$-Nitrobenzoylated alcohol $\mathbf{2 k}$ ' was purified by silicagel column chromatography (EtOAc/hexane 1:50) as a white solid ( $85 \%$ yield for 2 steps, $73 \%$ ee). ${ }^{1} \mathrm{H}$ NMR (300 MHz, $\left.\mathrm{CDCl}_{3}\right) \delta 8.36-8.31(\mathrm{~m}, 2 \mathrm{H}), 8.20-8.16(\mathrm{~m}, 2 \mathrm{H}), 4.36-4.29(\mathrm{~m}$, 2H), $2.08(\mathrm{~s}, 3 \mathrm{H}), 1.28(\mathrm{t}, 3 \mathrm{H}, J=7.3 \mathrm{~Hz}) ;{ }^{13} \mathrm{C} \mathrm{NMR}(75 \mathrm{MHz}$, $\left.\mathrm{CDCl}_{3}\right) \delta 164.3,162.4,151.2,134.3,131.2,123.9,118.4-104.7$ $(\mathrm{m}), 117.3\left(\mathrm{qt}, J_{\mathrm{C}-\mathrm{F}}=288.4,33.2 \mathrm{~Hz}\right), 82.8\left(\mathrm{t}, \mathrm{J}_{\mathrm{C}-\mathrm{F}}=25.4 \mathrm{~Hz}\right)$, $63.5,17.1,13.8 ;{ }^{19} \mathrm{~F}$ NMR $\left(282 \mathrm{MHz}, \mathrm{CDCl}_{3}\right) \delta-80.7$ (m, 3F), -116.4 to $-126.0(\mathrm{~m}, 14 \mathrm{~F})$; HRMS (APCI-TOF): $[\mathrm{M}]^{--}$calcd for $\mathrm{C}_{20} \mathrm{H}_{12} \mathrm{~F}_{17} \mathrm{NO}_{6}, 685.0393$; found, 685.0362; FTIR ( $\mathrm{KBr}$ pellet $\mathrm{cm}^{-1}$ ) 847, 969, 1009, 1142, 1214, 1246, 1297, 1472, 1530, 1613, 1732, 1757, 2339, 2360, 2860, 2922, 2997, 3112, 3454, 3493; $[\alpha]_{\mathrm{D}}{ }^{22}-11.93\left(c 0.48, \mathrm{CHCl}_{3}\right)$; HPLC (column, CHIRALPAK AD-3 and AD-H, hexane/2-propanol 99.5:0.5, flow rate $0.6 \mathrm{~mL} / \mathrm{min}, 20{ }^{\circ} \mathrm{C}$ detection UV $254 \mathrm{~nm}$ ) $t_{\mathrm{R}}$ of major isomer $16.8 \mathrm{~min}, t_{\mathrm{R}}$ of minor isomer $24.4 \mathrm{~min}$.

\section{Supporting Information}

\section{Supporting Information File 1}

Experimental details and characterization data of new compounds with copies of ${ }^{1} \mathrm{H},{ }^{13} \mathrm{C}$ and ${ }^{19} \mathrm{~F}$ NMR spectra. [https://www.beilstein-journals.org/bjoc/content/ supplementary/1860-5397-14-44-S1.pdf]

\section{Acknowledgements}

This work was financially supported by JST (ACT-C: Advanced Catalytic Transformation program for Carbon utilization), JSPS KAKENHI Grant Number 25410036, and the 
Noguchi Institute. We thank Central Glass Co., Ltd. for the gift of ethyl trifluoropyruvate (1a).

\section{References}

1. Müller, K.; Faeh, C.; Diederich, F. Science 2007, 317, 1881-1886. doi:10.1126/science.1131943

2. Hagmann, W. K. J. Med. Chem. 2008, 51, 4359-4369. doi:10.1021/jm800219f

3. Kirsch, P. Modern Fluoroorganic Chemistry: Synthesis, Reactivity, Applications, 2nd ed.; Wiley-VCH: Weinheim, Germany, 2013. doi:10.1002/9783527651351

4. Tomashenko, O. A.; Grushin, V. V. Chem. Rev. 2011, 111, 4475-4521. doi:10.1021/cr1004293

5. Liang, T.; Neumann, C. N.; Ritter, T. Angew. Chem., Int. Ed. 2013, 52, 8214-8264. doi:10.1002/anie.201206566

6. Sugiishi, T.; Amii, H.; Aikawa, K.; Mikami, K. Beilstein J. Org. Chem. 2015, 11, 2661-2670. doi:10.3762/bjoc.11.286

7. Nie, J.; Guo, H.-C.; Cahard, D.; Ma, J.-A. Chem. Rev. 2011, 111, 455-529. doi:10.1021/cr100166a

8. Mikami, K.; Itoh, Y.; Yamanaka, M. Chem. Rev. 2004, 104, 1-16. doi:10.1021/cr030685w

9. Gathergood, N.; Zhuang, W.; Jørgensen, K. A. J. Am. Chem. Soc. 2000, 122, 12517-12522. doi:10.1021/ja002593j

10. Zhuang, W.; Gathergood, N.; Hazell, R. G.; Jørgensen, K. A. J. Org. Chem. 2001, 66, 1009-1013. doi:10.1021/jo001176m

11. Török, B.; Abid, M.; London, G.; Esquibel, J.; Török, M.; Mhadgut, S. C.; Yan, P.; Prakash, G. K. S. Angew. Chem., Int. Ed. 2005, 44, 3086-3089. doi:10.1002/anie.200462877

12. Suri, J. T.; Mitsumori, S.; Albertshofer, K.; Tanaka, F.; Barbas, C. F., III. J. Org. Chem. 2006, 71, 3822-3828. doi:10.1021/jo0602017

13. Doherty, S.; Knight, J. G.; Smyth, C. H.; Harrington, R. W.; Clegg, W. J. Org. Chem. 2006, 71, 9751-9764. doi:10.1021/jo062023n

14. Rueping, M.; Theissmann, T.; Kuenkel, A.; Koenigs, R. M. Angew. Chem., Int. Ed. 2008, 47, 6798-6801. doi:10.1002/anie.200802139

15. Zhao, J.-F.; Tjan, T.-B. W.; Tan, B.-H.; Loh, T.-P. Org. Lett. 2009, 11, 5714-5716. doi:10.1021/ol902507x

16. Ogawa, S.; lida, N.; Tokunaga, E.; Shiro, M.; Shibata, N. Chem. - Eur. J. 2010, 16, 7090-7095. doi:10.1002/chem.201000911

17. Ohshima, T.; Kawabata, T.; Takeuchi, Y.; Kakinuma, T.; Iwasaki, T.; Yonezawa, T.; Murakami, H.; Nishiyama, H.; Mashima, K. Angew. Chem., Int. Ed. 2011, 50, 6296-6300. doi:10.1002/anie.201100252

18. Dong, X.; Sun, J. Org. Lett. 2014, 16, 2450-2453. doi:10.1021/ol500830a

19. Morisaki, K.; Morimoto, H.; Mashima, K.; Ohshima, T. Heterocycles 2017, 95, 637-661. doi:10.3987/REV-16-SR(S)4

20. Aikawa, K.; Kainuma, S.; Hatano, M.; Mikami, K. Tetrahedron Lett. 2004, 45, 183-185. doi:10.1016/j.tetlet.2003.10.137

21. Mikami, K.; Kawakami, Y.; Akiyama, K.; Aikawa, K. J. Am. Chem. Soc. 2007, 129, 12950-12951. doi:10.1021/ja076539f

22. Aikawa, K.; Hioki, Y.; Mikami, K. Org. Lett. 2010, 12, 5716-5719. doi:10.1021/ol102541s

23. Mikami, K.; Aikawa, K.; Aida, J. Synlett 2011, 2719-2724. doi:10.1055/s-0031-1289540

24. Aikawa, K.; Hioki, Y.; Shimizu, N.; Mikami, K. J. Am. Chem. Soc. 2011, 133, 20092-20095. doi:10.1021/ja2085299

25. Aikawa, K.; Asai, Y.; Hioki, Y.; Mikami, K. Tetrahedron: Asymmetry 2014, 25, 1104-1115. doi:10.1016/j.tetasy.2014.06.013
26. Aikawa, K.; Kondo, D.; Honda, K.; Mikami, K. Chem. - Eur. J. 2015, 21, 17565-17569. doi:10.1002/chem.201503631

27. Aikawa, K.; Yoshida, S.; Kondo, D.; Asai, Y.; Mikami, K. Org. Lett. 2015, 17, 5108-5111. doi:10.1021/acs.orglett.5b02617

28. Aicher, T. D.; Anderson, R. C.; Bebernitz, G. R.; Coppola, G. M.; Jewell, C. F.; Knorr, D. C.; Liu, C.; Sperbeck, D. M.; Brand, L. J.; Strohschein, R. J.; Gao, J.; Vinluan, C. C.; Shetty, S. S.; Dragland, C.; Kaplan, E. L.; DelGrande, D.; Islam, A.; Liu, X.; Lozito, R. J.; Maniara, W. M.; Walter, R. E.; Mann, W. R. J. Med. Chem. 1999, 42, 2741-2746. doi:10.1021/jm9902584

29. Aicher, T. D.; Anderson, R. C.; Gao, J.; Shetty, S. S.; Coppola, G. M.; Stanton, J. L.; Knorr, D. C.; Sperbeck, D. M.; Brand, L. J.; Vinluan, C. C.; Kaplan, E. L.; Dragland, C. J.; Tomaselli, H. C.; Islam, A.; Lozito, R. J.; Liu, X.; Maniara, W. M.; Fillers, W. S.; DelGrande, D.; Walter, R. E.; Mann, W. R. J. Med. Chem. 2000, 43, 236-249. doi:10.1021/jm990358+

30. Bebernitz, G. R.; Aicher, T. D.; Stanton, J. L.; Gao, J.; Shetty, S. S.; Knorr, D. C.; Strohschein, R. J.; Tan, J.; Brand, L. J.; Liu, C.; Wang, W. H.; Vinluan, C. C.; Kaplan, E. L.; Dragland, C. J.; DelGrande, D.; Islam, A.; Lozito, R. J.; Liu, X.; Maniara, W. M.; Mann, W. R. J. Med. Chem. 2000, 43, 2248-2257. doi:10.1021/jm0000923

31. Gosselin, F.; Britton, R. A.; Mowat, J.; O’Shea, P. D.; Davies, I. W. Synlett 2007, 2193-2196. doi:10.1055/s-2007-984911

32. Konigsberger, K.; Prasad, K.; Repič, O. Tetrahedron: Asymmetry 1999, 10, 679-687. doi:10.1016/S0957-4166(99)00017-8

\section{License and Terms}

This is an Open Access article under the terms of the Creative Commons Attribution License (http://creativecommons.org/licenses/by/4.0), which permits unrestricted use, distribution, and reproduction in any medium, provided the original work is properly cited.

The license is subject to the Beilstein Journal of Organic Chemistry terms and conditions: (https://www.beilstein-journals.org/bjoc)

The definitive version of this article is the electronic one which can be found at: $\underline{\text { doi: } 10.3762 / \text { bjoc. } 14.44}$ 